\title{
Multilokuläre adulte Rhabdomyome als seltene Ursache einer Dysphagie
}

\author{
P. Isfort, M. Grosheva, A.I. Schulz, K.-B. Hüttenbrink, D. Beutner
}

\begin{tabular}{|l|}
\hline \multicolumn{2}{|c|}{ Einleitung } \\
\hline Die Differentialdiagnosen einer Dysphagie umfassen neben \\
neurologischen Ursachen auch mechanische Behinderungen wie \\
benigne oder maligne Raumforderungen, sowie Anomalien. \\
Adulte Rhabdomyome sind seltene benigne Tumore der Herz- \\
und quergestreiften Muskulatur. Extrakardiale adulte \\
Rhabdomyome im Kopf- und Halsbereich sind sehr selten. Das \\
multilokuläre Auftreten bleibt eine Rarität. \\
\hline
\end{tabular}

\begin{tabular}{|l|}
\hline Kasuistik \\
\hline Wir berichten über einen 45-jährigen Patienten, der unter einer \\
progredienten Dysphagie seit 6 Monaten litt. Klinisch imponierte \\
eine submuköse Raumforderung im Bereich der rechten \\
Rachenhinterwand, sowie eine kontralaterale weiche Neubildung \\
links zervikal. Die histologische Aufarbeitung der vollständig \\
exstirpierten Tumore erbrachte die Diagnose eines multilokuären \\
adulten Rhabdomyoms. \\
Der postoperative Verlauf war komplikationslos. Der Patient war \\
bei einer Wiedervorstellung nach 4 Wochen beschwerdefrei. \\
\hline
\end{tabular}
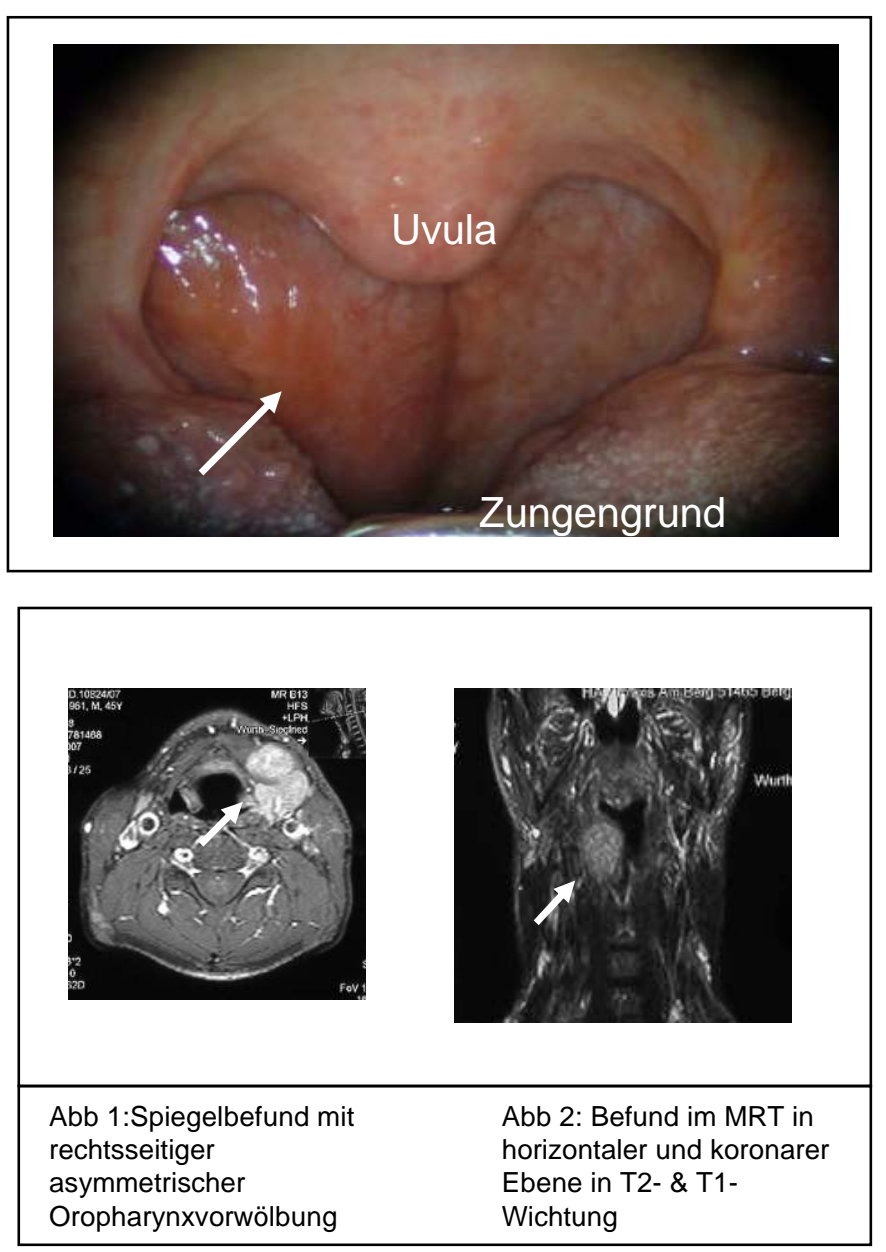

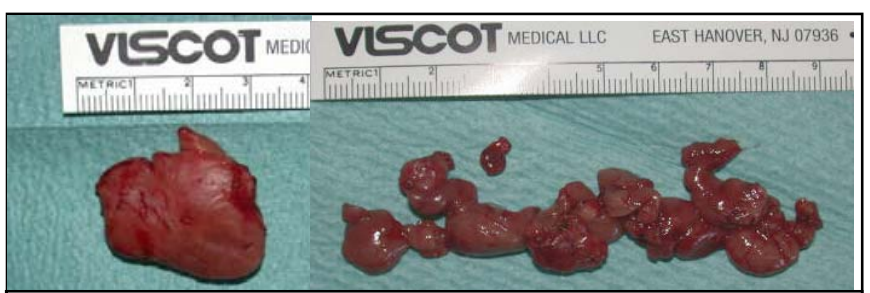

Abb. 3: grosses solitäres und multiple kleine Rhabdomyom-Fragmente

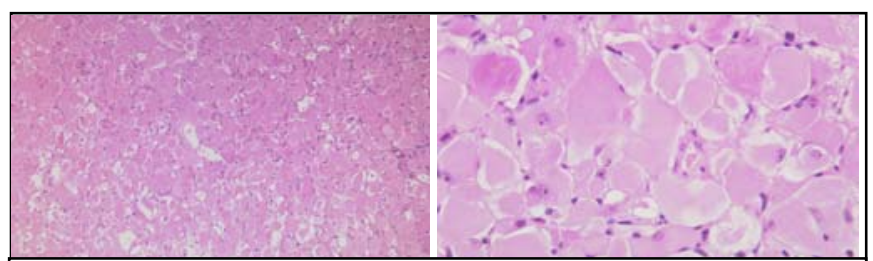

Abb. 4: Lichtmikroskopischer Aspekt in Übersichts- \& stärkerer Vergrösserung

(1)

Eine retropharyngeale Raumforderung ist keine seltene Ursache einer Dysphagie. Differentialdiagnostisch müssen immer auch maligne Raumforderungen des Kopf- und Halsbereichs, sowie des Ösphagus und dessen angrenzenden Strukturen bedacht werden. Ausserdem gibt es ein weites Spektrum benigner Ursachen einer Dysphagie.

Adulte extrakardiale Rhabdomyome sind seltene gutartige Tumoren, die sich am häufigsten im Kopf-Hals-Bereich manifestieren. Sie wachsen in des meisten Fällen (70\%) solitär und treten häufiger im höheren Lebensalter und bei Männern auf (medianes Alter: 60 Jahre; $\mathrm{m}: \mathrm{f}=3: 1$ ). Sie sind neben der o.g. Lokalisation noch im Larynx, parapharyngeal, i.B.d. Zunge, des Zungengrundes, der Wange und der Lippen anzutreffen. Sehr selten trifft man sie an den Extremitäten, im Ösophagus, im Magen, im Mediastinum, in der Orbita, in der Prostata und intrakraniell an.

Die Therapie der Wahl ist die Exzision in toto. Die Inzidenz von Rezidiven wird in der Literatur mit bis zu 42\% angegeben. Als Ursache hierfür wird eine initiale inkomplette Resektion angesehen. Eine maligne Transformation wurde noch nicht beschrieben.

\section{Schlussfolgerung}

Adulte multilokuläre Rhabdomyome, als seltene benigne Läsionen im Kopf - Hals - Bereich, können durch ihr klinisches Bild einen metastasierenden malignen Prozess vortäuschen und die Wahl des therapeutischen Vorgehens erschweren. Die vollständige Entfernung des Tumors ist essenziell, um Lokalrezidive zu vermeiden.

Kontakt: Dr. med. P.Isfort, HNO-Klinik der Universität zu Köln, Kerpener Str. 62, 50924 Köln

Literatur: Bitte Anfrage an Autor: peter.isfort@uk-koeln.de 